\title{
Intrathecal Use of Amikacin: A Case Report
}

\author{
L. Barrou, B. Charra, A. Hachimi, B. Idali, A. Benslama and S. Motaouakkil \\ Reanimation Anaesthesia Department, Ibn Rochd University Hospital; Casablanca, Morocco
}

\begin{abstract}
Meningitis caused by Acinetobacter baumannii is rare and are mostly hospital acquired after neurosurgical procedure. We report a case of a 40 -year old man was admitted to the intensive care unit due to subarachnoid haemorrhage. Our patient developed a ventriculitis due to A.baumannii treated successfully with sulbactam IV and intrathecal amikacin. Key-Words: Amikacin, intrathecal, nosocomial meningitis.
\end{abstract}

Meningitis caused by Acinetobacter baumannii is rare and are mostly hospital acquired after neurosurgical procedure [1]. The penetration of aminoglycosides to cerebrospinal fluid (CSF) is poor. There are several reports about intrathecal (IT) administration of gentamicin; the highest reported IT aminoglycoside dose is $100 \mathrm{mg}$ amikacin [2].

\section{Case Report}

A 40-year old man was admitted to the intensive care unit with subarachnoid haemorrhage. The computerized tomography scan showed intraventricular bleeding, dilation of the lateral ventricles, and a decrease in size of the fourth ventricle. The patient underwent a craniotomy for clipping of an aneurysm and we performed an external drainage.

The patient developed fever and his general status deteriorated 8 days after the drainage. CSF protein level was $0,55 \mathrm{~g} / \mathrm{L}$, glucose level was $0,38 \mathrm{~g} / \mathrm{L}$ (blood glucose $=0,98 \mathrm{~g} /$ L). Microscopic examination of CSF revealed 650 leukocytes/ $\mathrm{mm}^{3}$ (80\% PNL) but no specific organism was seen in Gramstain. His WBC count was $15,500 / \mathrm{mm}^{3}$ (90\% PNL), creatinin level was $0,45 \mathrm{mg} / \mathrm{L}$. Despite therapy with intravenous (IV) amikacin 1,000 mg and imipenem 500mg every 4 hours, the infection persisted. The CSF culture yielded A. baumannii which was sensitive to sulbactam, amikacin, imipenem, ciprofloxacin and gentamicin by disc diffusion method. Imipenem/cilastatin and IV amikacin were stopped and IV sulbactam $3 g$ twice daily was initiated. Intrathecal amikacin 40 mg daily was added. The ventriculostomy drain was clamped for 1 hour following each IT infusion. CSF amikacin concentration $1 \mathrm{~h}$ after infusion exceeded $100 \mu \mathrm{g} / \mathrm{mL}$. One week after IT amikacin treatment no growth was detected in CSF culture and no leukocytes were seen on microscopy. On the $10^{\text {th }}$ day of the IT therapy patient's status deteriorated and he developed fever $\left(39^{\circ} \mathrm{C}\right)$. Enterococcus faecium was grown from blood culture. IV Vancomycin 1,500 mg (qid) was added but patient was lost 2 days later. CSF sample, obtained on the day he died, was sterile and contained 10 leukocytes $/ \mathrm{mm}^{3}$.

\section{Discussion}

Several authors have reported on the use of IT amikacin at various doses for patients with Gram-negative bacillary meningitis. Ngyen reported on the use of intravenous therapy in combination with IT aminoglycosides for postneurosurgical Acinetobacter meningitis [3].

Therapy was successful in two of three patients treated with IT gentamicin one of two patients treated with IT amikacin. In limited case reports radicular pain, meningeal irritation, seizures and ototoxicity have been reported as potential adverse reactions to IT amikacin. The maximal tolerable dose of IT amikacin is not known, but some patients have received up to $40 \mathrm{mg} /$ day without apparent toxicity [4]. In the case presented we used $40 \mathrm{mg}$. His severe neurological impairment due to the underlying illness significantly limited our ability to detect CNS toxicity.

\section{References}

1. Kendirli T., Aydin H.I., Hacihamdioglu D., et al. Meningitis with multidrug-resistant Acinetobacter baumanii treated ampicillin /sulbactam. J Hosp Infect 2004;56:328.

2. Yamashima T., Shoin K., Someya S., et al. Intrathecal administration of amikacin in postoperative refractory meningitis. Jpn J Antibiot 1983;36:522-8.

3. Berning S.E., Cherry T.A., Iseman M.D. Novel treatment of meningitis caused by multidrug-resistant Mycobacterium tuberculosis with intrathecal levofloxacin and amikacin: case report. Clin Infect Dis 2001;32(4):643-6.

4. Gilbert V.E., Beals Jr. J.D., Natelson S.E., Tyler W.A. Treatement of cerebrospinal fluid leaks and Gram-negative bacillary meningitis with large doses of intrathecal amikacin and systemic antibiotics. Neurosurgery 1986;18(4):402-6.

Received on 6 October 2008; revised 27 November 2008.

Address for correspondence: Dr. Said Motaouakkil. Ibn Rochd University Hospital, 1, quartier des hôpitaux, Casablanca 20 100. Morocco. Phone : $0021261 \quad 1553$ 11. E-mail : smotaouakkil@yahoo.fr/reamedcasa@gmail.com. 